\title{
Sensor-based Experimental Evaluation of Mixing Characteristics in Laboratory- scale Reactor Systems
}

\section{Dr. Steven C. Chiesa P.E., Santa Clara University}

Professor Chiesa is an associate professor in the Department of Civil Engineering at Santa Clara University. He has been teaching environmental engineering courses at the university level for over 30 years. He holds a BS degree from Santa Clara University, an MS degree from Stanford University, and a PhD degree from the University of Notre Dame. 


\section{Sensor-based Experimental Evaluation of Mixing Characteristics in Laboratory-scale Reactor Systems}

\section{Introduction}

Environmental engineering has increasingly integrated concepts from chemistry, microbiology, and chemical engineering to explain and model both natural and engineered systems. Reaction engineering fundamentals are typically introduced as part of an initial undergraduate course in environmental engineering to help provide a framework for relevant mass balance applications. In such cases, idealized mixing conditions can be used to develop behavior/performance relationships for simple batch (fill-and-draw) systems as well as continuous flow complete-mix and ideal plug flow reactor configurations. Subsequent coursework can be used to provide follow-up discussions of how such idealized system models can be modified and/or combined for use in understanding and modeling non-ideal reactor system behavior.

One of the more traditional ways of identifying reactor system mixing characteristics is the use of tracer studies. The response in system effluent tracer concentration with time after a pulse (slug) or a step increase in the system influent tracer concentration can be used to determine how closely a given reactor system follows one of the idealized system responses (Viessman et al., 2008; Davis and Cornwell, 2013 ). Mathematical modeling based on (dynamic) mass balance concepts and/or a statistical analysis using the tracer effluent-versus-time concentration data could then be used to provide evidence in supporting the degree of conformity to idealized behavior. To help reinforce the ability of tracer concentration response patterns to identify reactor mixing behavior, a hands-on laboratory experiment was designed and implemented to evaluate the ability of a sensor-responsive dye tracer to characterize the mixing conditions in laboratory-scale reactor systems. The sensor and its integrated data acquisition and analysis system were chosen to provide continuous data tracking and reduce the need for more traditional wet-chemistry-based techniques for data collection. Development of the test system required adapting the Turner Designs Cyclops ${ }^{\mathrm{TM}}$ Rhodamine-specific sensor and its companion Explorer $^{\mathrm{TM}}$ data acquisition module for use at lab scale. Experimentation required small student groups to configure system components according to a schematic diagram, establish and document steady-state hydraulic behavior for the system, and then generate a set of data chronicling the system's response to a pulse input of Rhodamine dye tracer. Data were used to compare actual and theoretical (ideal) system responses to develop conclusions as to the mixing behavior of the system.

The student learning outcomes chosen for this experimental work addressed concepts related to conducting experiments and analyzing/interpreting data (ABET Student Outcome 3b) and using techniques, skills, and modern analytical tools (ABET Student Outcome 3k). Because of the need to use small student teams to complete the experimentation phase, the metric developed to assess student learning includes several elements related to how well the team conducted the experimental work. The larger fraction of the assessment metric, however, focusses on individual student learning as evidenced by the content of a submitted written laboratory report. 


\section{Experimental Set-up}

The experimental system consists of a feed (tap) water reservoir, pump, the chosen test reactor system, the dye sensor and associated flow-through cell, the companion data acquisition module, and a personal computer containing the data acquisition software. Figure 1 schematically depicts the system set up in its simplest configuration using a single, mixed reaction vessel. The plastic reaction vessel has a maximum working volume of 3.785 liters and is clear-walled to provide easy visual observation of the changes in tracer concentration as the dye works its way through the system. The adjustable PVC pipe-based overflow system is used to control the active working volume of the test reactor at a pre-determined level.

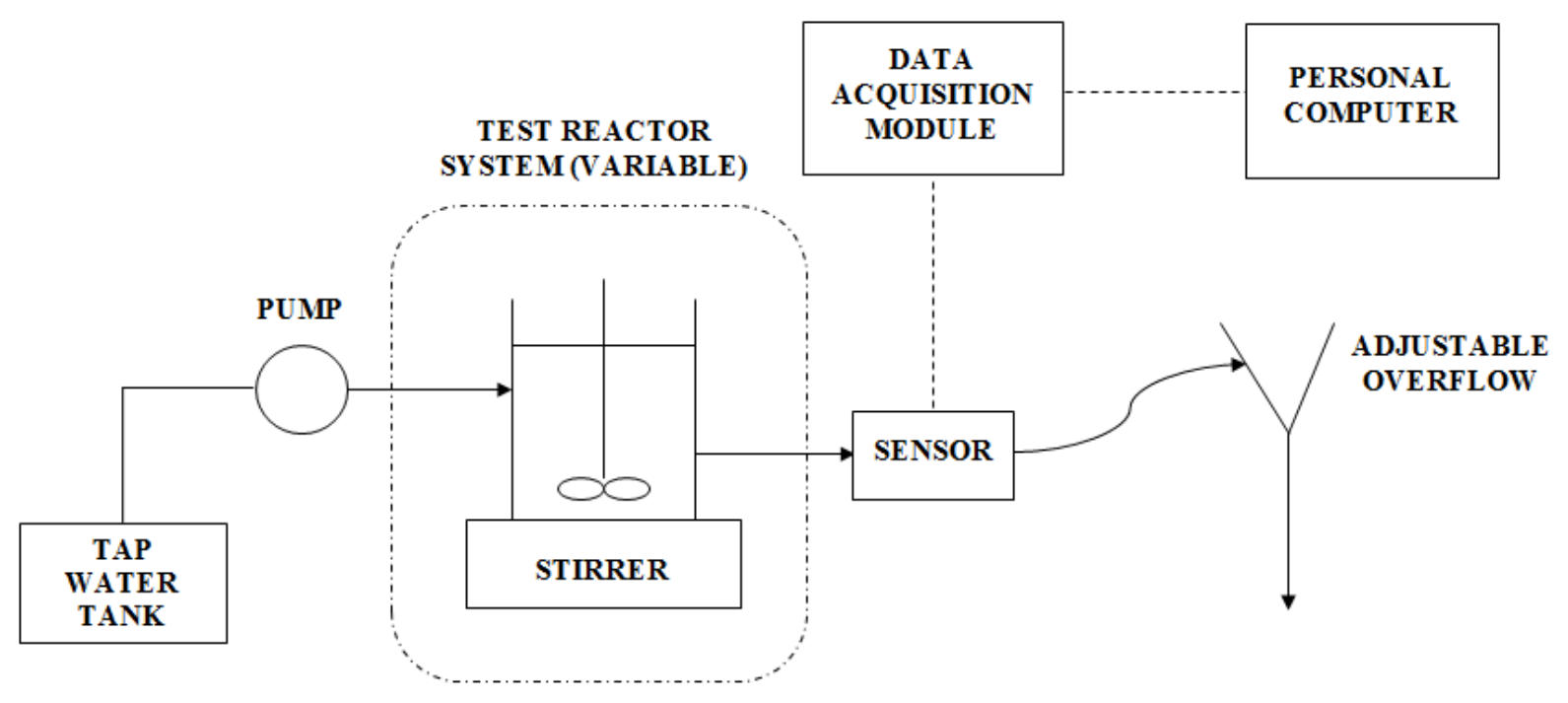

Figure 1. Test System Schematic Diagram

Experiments currently have students using variable speed peristaltic pumps to provide an appropriate, constant volumetric flow rate. Volumetric flow rates are set at different values depending on the size and hydraulic complexity of the test reactor system being evaluated. The flow rate for the aforementioned single reactor system is maintained at approximately 0.55 liters per minute to limit hydraulic headlosses and to complete the operational phase of the experiment in less than 30 minutes. Mixing was provided using "floating" stir bars and variable speed magnetic stirrers to better control mixing intensity. Mixing intensity was set to provide a slight vortex on the surface of the reactor. Flexible plastic tubing has been used to connect system components with tubing lengths minimized whenever possible. Black Viton ${ }^{\mathrm{TM}}$ tubing has been used for sensor inlet and outlet connections to eliminate possible interference.

The Turner Designs Cyclops ${ }^{\mathrm{TM}}$ and its associated Explorer ${ }^{\mathrm{TM}}$ data acquisition system were chosen for several reasons. The sensor and data acquisition system is primarily marketed for full-scale field studies but is also fortuitously available with a flow-through cell attachment for small-scale applications. The small size of the sensor (approximately six inches long) allows it to be easily configured for lab-scale use. The sensor exhibits an extensive linear response range when used with Rhodamine dye. Three optional sensitivity modes allow the sensor to be used 
with the relatively high, easily visible dye concentrations used in these experiments. Sensor output is provided in VDC (or mVDC) units but these values can be converted to concentration values if VDC units cannot be used as a surrogate measure in subsequent data reduction and analysis operations. The Explorer ${ }^{\mathrm{TM}}$ data acquisition software allows sensor readings to be taken either at any user-selected time or automatically at a user-defined interval. Recorded data can be saved and exported in several different file formats for subsequent analysis using spreadsheet programs. The Cyclops ${ }^{\mathrm{TM}}$ sensor was actually used in these experiments for several years before the data acquisition system was introduced. With no data acquisition system, a multimeter was used to measure sensor output with the VDC-versus-time output data was recorded manually. The multimeter read-out option requires the use of a DC power supply to drive the sensor.

A photograph showing the Cyclops ${ }^{\mathrm{TM}}$ sensor with and without its flow-through cell is provided in Figure 2. A photograph of the sensor, as installed in the basic test system, is provided in Figure 3. Before any reactor-based mixing experimentation was conducted, the sensor was tested to ensure a consistent linear relation between net (after background correction) sensor output and dye concentration. Dye concentrations up to levels well in excess of those anticipated during actual system testing were used to verify the linear response range of the sensor.

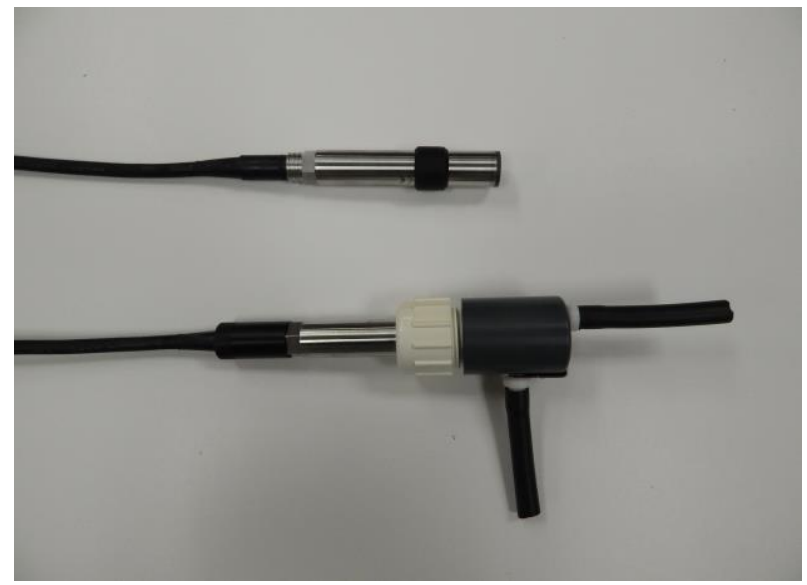

Figure 2. Sensor and Sensor with Flow Cell

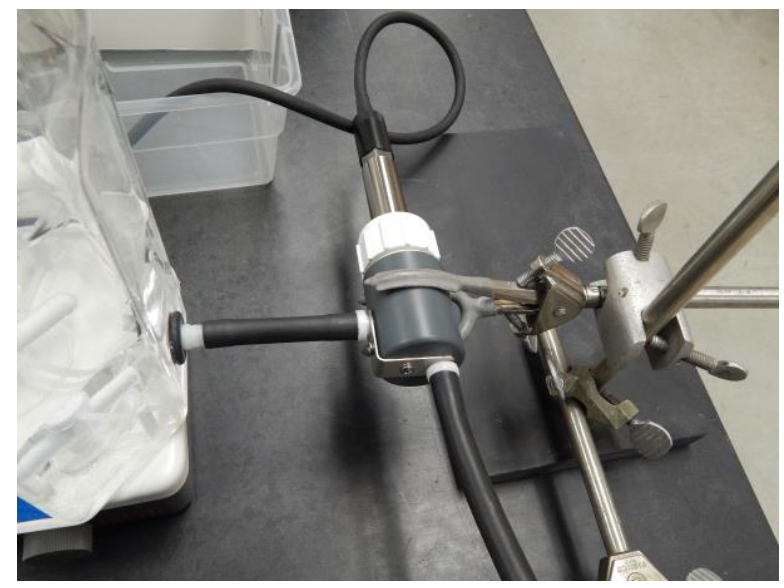

Figure 3. Sensor Installed in a Test System

After assembly of the system has been completed and checked, the pump in turned on and reactor system operation is initiated with tap water. The system is allowed time to reach a steady state hydraulic condition as evidenced by a constant target liquid volume in the test reactor system. Achieving this target liquid volume requires systematically adjusting the overflow device and allowing the system to quickly re-equilibrate. Once steady state hydraulic conditions are realized, the data acquisition system is activated and set to provide readings at a specified interval (typically one minute). System initial response readings are then recorded to document the background sensor response to tap water. Dye tracer studies have focused on the use of slug/pulse inputs of Rhodamine dye after the system has achieved hydraulic equilibrium. The most convenient, rapid method for introducing dye into the first mixed chamber of the test system has involved using a calibrated syringe filled to a pre-determined level with the Rhodamine dye solution. Attempts to introduce the dye slug directly into the influent tap water feed tubing have not been successful as some small amount of dye appears to leak from the syringe tip into the injection port before the designated injection time. Injection of the dye 
requires close coordination with the programmed sensor reading interval; the dye must be injected into the test system immediately after the last background sensor reading has been recorded. After the tracer is injected, the system operates on its own for enough time to gather sufficient data to determine reactor mixing conditions. Figures 4 depicts a single reactor test system with a 2.0 liter working volume after dye injection. Figure 5 provides a screen shot of the data acquisition system later during this same test.

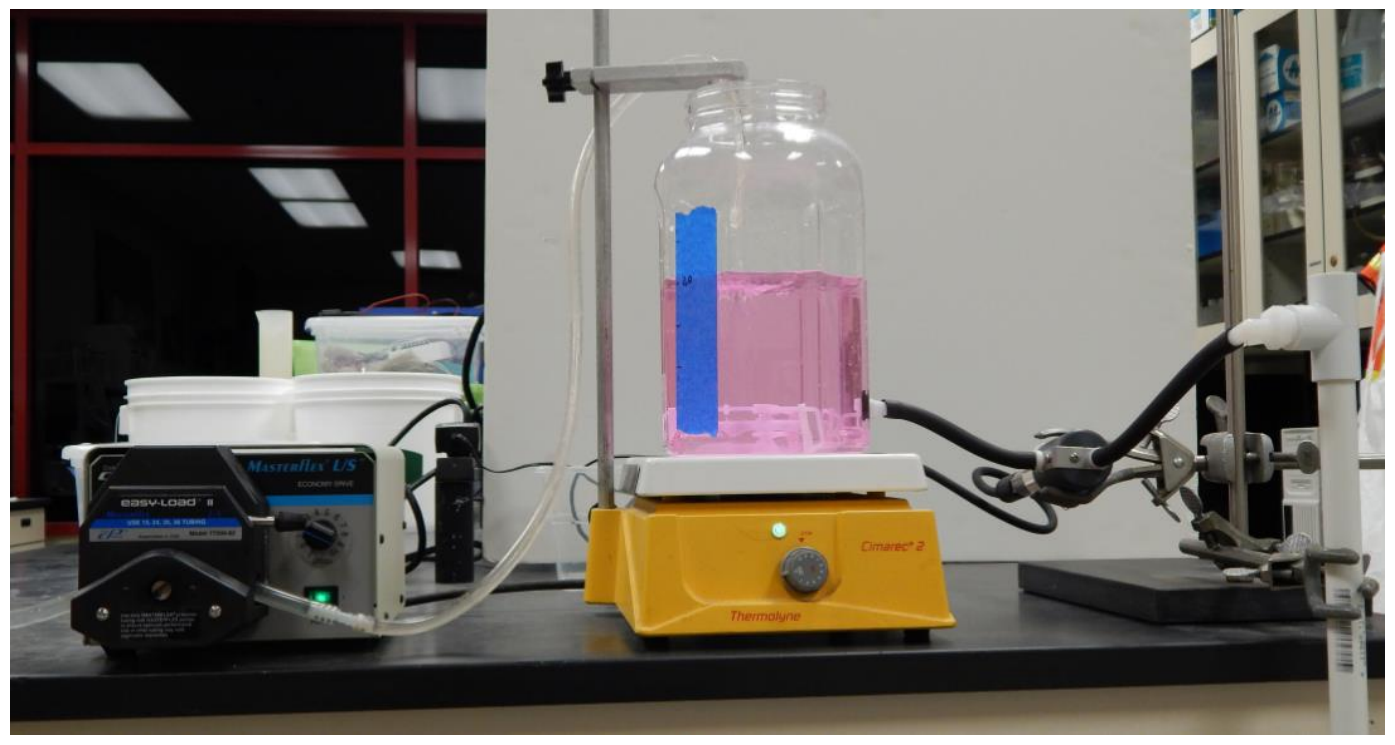

Figure 4. Single Reactor Dye Tracer Study During Operation

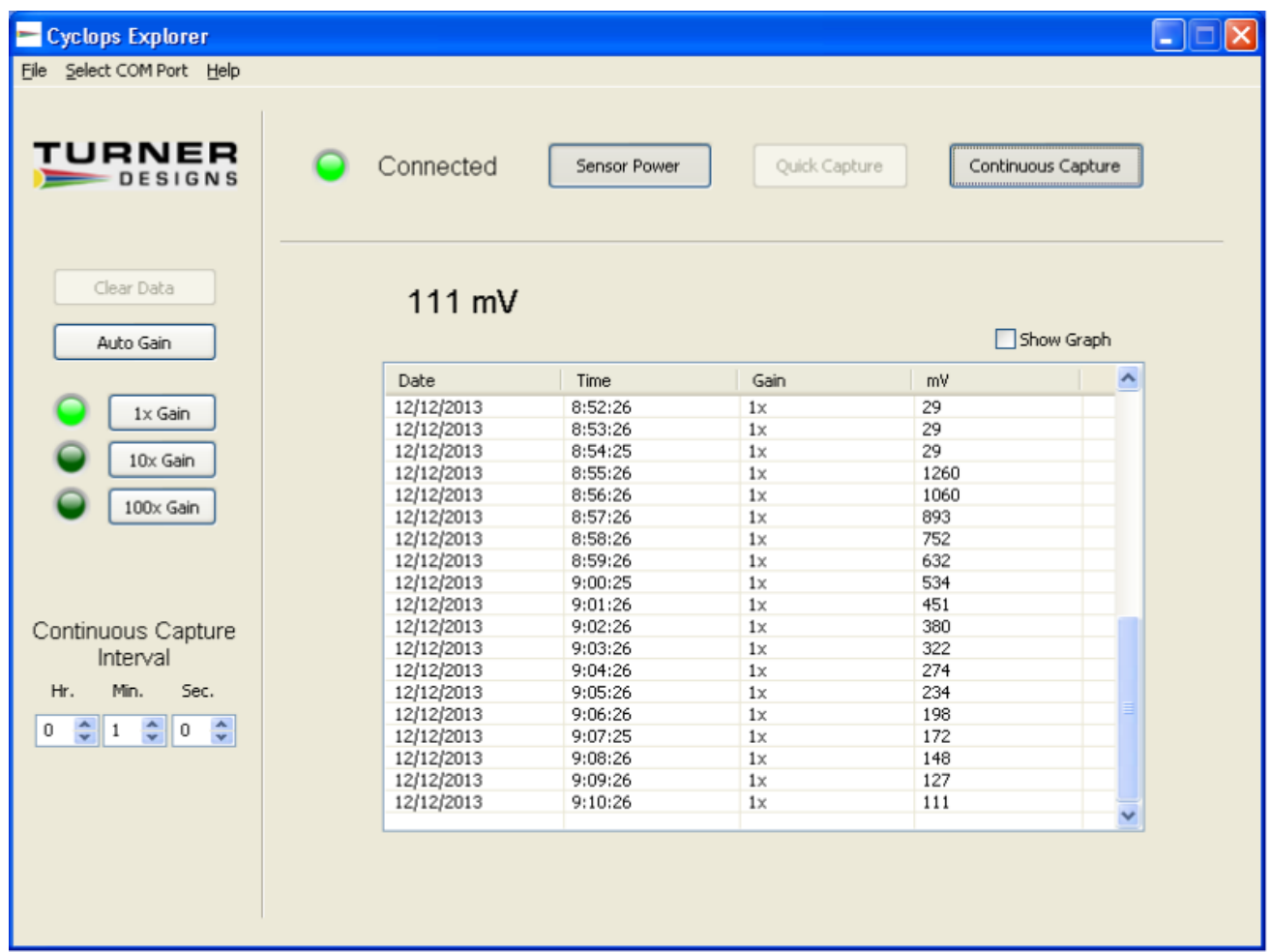

Figure 5. Data Acquisition System Output for the Single Reactor Dye Tracer Study 
The first three $29 \mathrm{mV}$ sensor output readings recorded in Figure 5 represent background readings with tap water passing through the test system and sensor. Dye was injected directly into the test system immediately after the third $29 \mathrm{mV}$ reading was taken. The $1260 \mathrm{mV}$ reading was recorded one minute after the dye injection. While the dye slug passes through the test system and effluent dye concentration levels are automatically recorded, student teams capture the system effluent flow for a designated period of time and use the captured fluid volume together with the associated elapsed capture time to determine the volumetric flow rate.

The dye sensor and companion data acquisition system can potentially be used with a variety of test experimental reactor systems that vary in both physical size and hydraulic complexity. As detailed earlier, experiments in the introductory environmental engineering course have focused on the response of a single continuous flow, completely mixed reactor. A laboratory experiment for a senior-level elective environmental reaction engineering course has been developed and used to investigate the dye tracer response from a test system comprised of four equally-sized, continuous flow, completely mixed reactors connected in series. Figure 6 portrays the four compartment system during such a dye tracer experiment. The lower volumetric flow rates needed to control cumulative headloss in the test system and longer overall hydraulic detention time have resulted in roughly two hour operational response periods for these test systems.

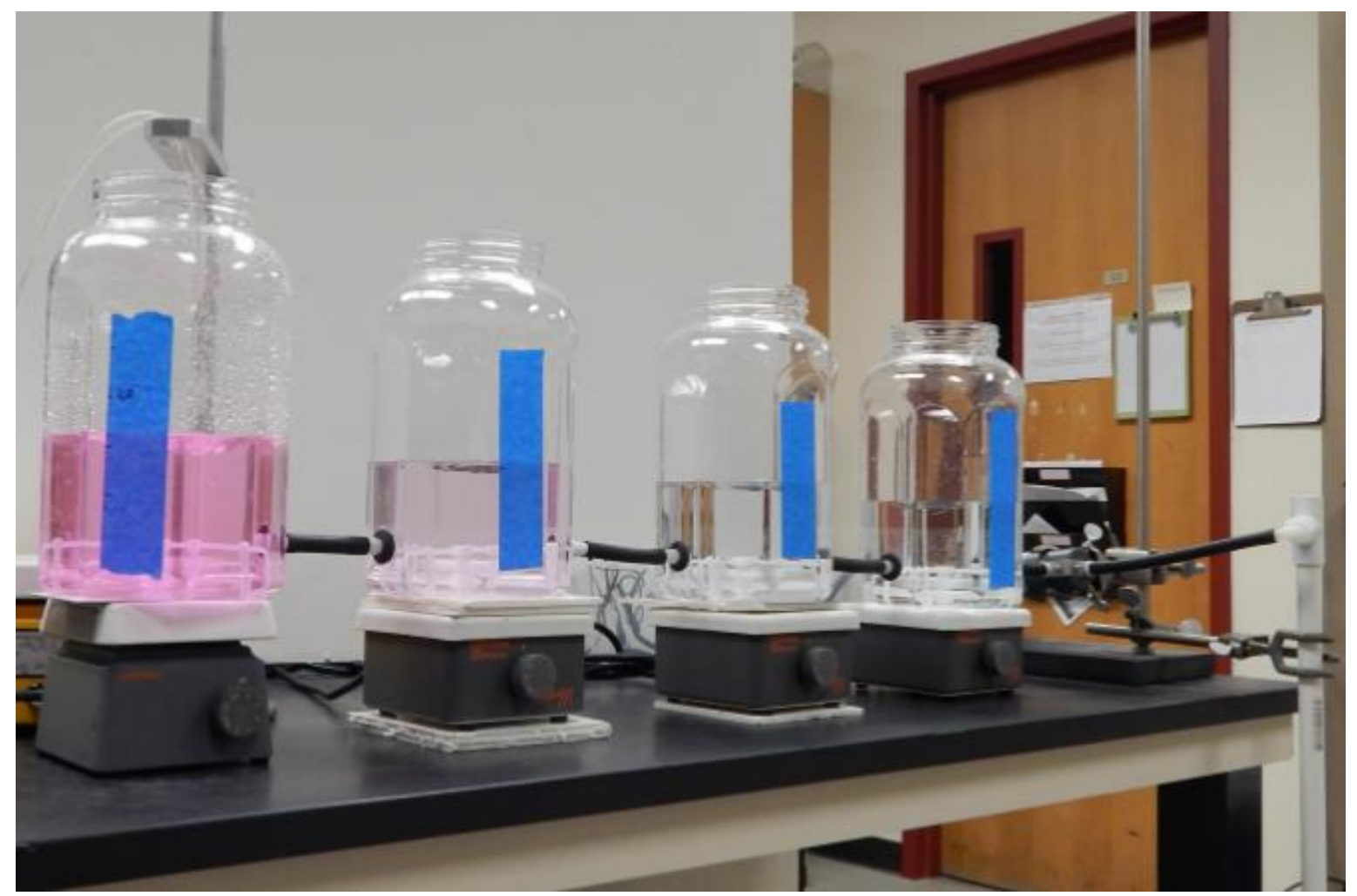

Figure 6. Sensor-based Dye Tracer Experiment with Four Mixed Reactors in Series.

To maintain equal, constant volumes in each of the four-1.50 liter mixed reactors, a combination of different magnetic stirrers and small, thin shims have been used to compensate for the hydraulic headlosses realized as flow passes through the system. The back-end dye sensor and effluent overflow/level control system configuration is identical for all test reactor systems. 


\section{Experimental Data Analysis and Laboratory Reporting Protocols}

As part of the laboratory report submitted for this exercise, students were instructed to analyze the experimental data and compare the actual tracer response with the response predicted by mass balance models developed for the associated ideal reactor system. For an ideal continuous flow, completely mixed reactor subjected to a slug tracer input, the theoretical effluent tracer concentration response would take the form of a simple exponential decay. Figure 7 depicts an example of actual data plotted together with a computer-generated best fit exponential decay response for these data. It should again be noted that the net sensor output readings are directly proportional to the effluent dye concentration and that the net sensor output readings represent the gross (measured) sensor values initially recorded reduced by the background sensor reading observed for the tap water being used.

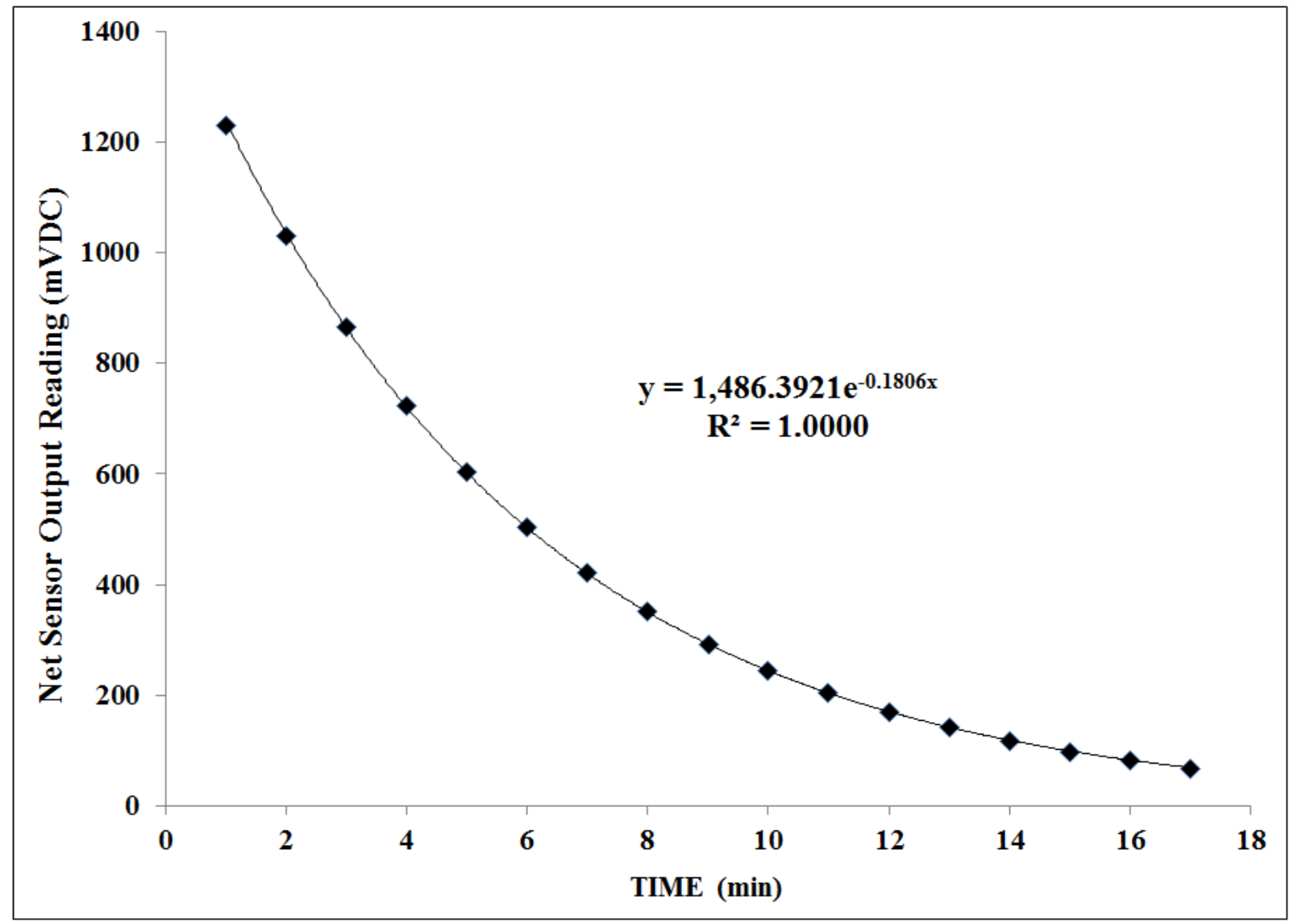

Figure 7. Comparison of Experimental Data with Theoretical Response for a Single Complete Mix Reactor $(\checkmark$ experimental data, $-=$ best-fit effluent response)

The numbers in the equation for the best-fit exponential decay relationship provided in Figure 7 were re-formatted to minimize potentially significant rounding errors associated with the default format. This is a key adjustment in reducing errors in follow-up student calculations. As an alternative to the data presentation format presented in Figure 7, students were given the option of providing a linearized version of the graphical response comparison by plotting natural log values of the net sensor output versus time. The two approaches provide equivalent information. 
The general exponential decay effluent tracer concentration response indicated in Figure 7 would be expected for any single continuous flow, completely mixed reactor, regardless of reactor working volume. To better relate the observed tracer response to the specific reactor system used in the experiment, students were asked to first determine the average hydraulic detention time for the reactor system using information derived from the best-fit exponential decay equation found as part of the associated spreadsheet-based data analysis (again assuming an ideal theoretical response to a slug input of tracer into a completely mixed system). Students were then instructed to calculate the system average hydraulic detention time using experimentally determined/measured values for the reactor liquid volume, V, and the volumetric flow rate, $\mathrm{Q}$, passing through the system. Parameters V and Q would necessarily be expressed in consistent and compatible units. Differences between the two calculated average detention time values could then be used as the focal point for an error analysis.

The same basic sensor and overflow system can be used to examine other, more complex experimental systems with the possible intent of comparing actual and theoretical system tracer responses, determining the system dispersion number, or estimating the extent of dead volume present in a system. The continuous flow system consisting of four equal volume, completely mixed reactors in series has been used to extend the concept of system mixing beyond just a single, well-mixed reactor. Figure 8 indicates actual and predicted system effluent tracer concentration responses for the four reactors in series system configuration.

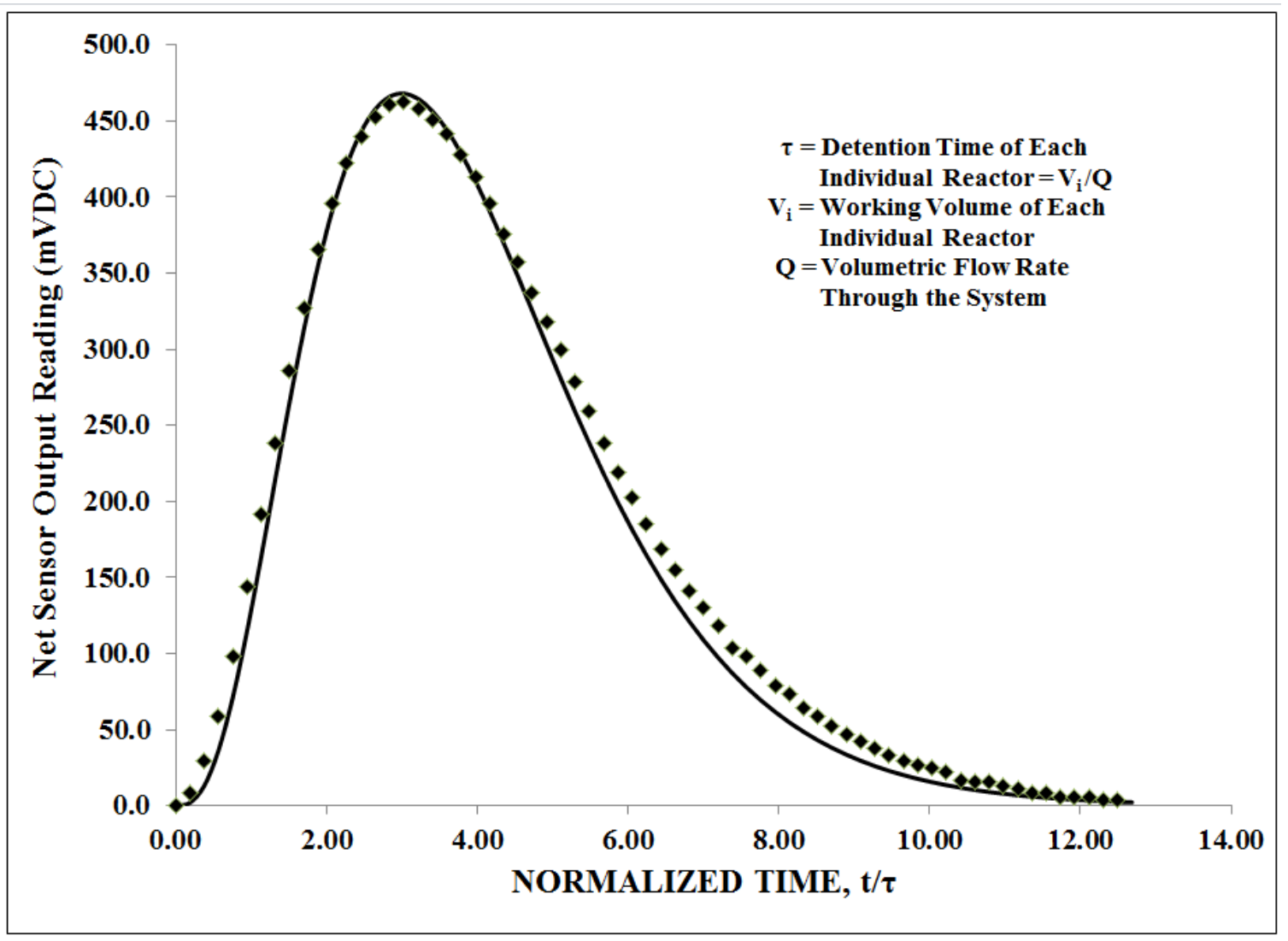

Figure 8. Comparison of Experimental Data with Theoretical Response for Four Equally-Sized Reactors in Series $(\diamond=$ experimental data, $-=$ theoretical effluent response) 
The theoretical effluent tracer concentration response-versus-time curve in Figure 8 was produced using a relationship reported by others ${ }^{1}$ for the last reactor in a multi-reactor series configuration of equally sized, completely mixed vessels. After gross (measured) sensor readings recorded by the Explorer ${ }^{\mathrm{TM}}$ data acquisition system, students are instructed to save the information in an appropriate file format and then transfer the raw data into a spreadsheet program. Students must then reduce the data by correcting for background sensor readings and then perform a statistical analysis of the reduced data to produce estimates for the system mean residence time and variance in the data distribution. The mean residence time and variance values can then be used by students to calculate the effective number of equal working volume reactors operating in series ${ }^{1}$. This calculated value can be compared with the actual number of vessels employed in the experiment as a means of evaluating the ability of the experimental system to provide ideal conditions. The same calculated mean residence time and variance values can also be used to estimate the longitudinal dispersion number for the reactor system ${ }^{2}$.

While the actual experimentation and associated data collection were organized as small group activities, students were required to individually prepare written laboratory reports with a specific list of deliverables. These student deliverables included answering a series of basic questions related to the underlying concepts behind the experimentation, summarizing the visual observation they made during the time the dye tracer passed through the system, providing an evaluation of the data in the context of the referenced ideal system behavior, and discussing potential sources of error and/or reasons behind and observed differences between actual and theoretical behavior.

\section{Assessment of Student Learning Outcomes}

Intended student learning goals for this laboratory exercise have focused on ABET-oriented outcomes for conducting experiments and analyzing/interpreting data (ABET Student Outcome 3b) and using techniques, skills, and modern analytical tools (ABET Student Outcome 3k). The nature of this particular experimentation did not allow for any meaningful experimental design component and this student learning outcome is being addressed elsewhere in the curriculum. Assessment of student learning involved preparing a simple rubric for evaluating how well students are able to address the objectives established for the laboratory exercise. The rubric was used as the key part of evaluating student work as lab work was being conducted and for work submitted with the associated written lab reports.

The rubric currently being used in the required introductory environmental engineering course using the single well-mixed reactor is provided in Table 1. After the first year that this experiment was used as part of the course, the rubric itself was included as part of the handout distributed several days before students conducted the experimental work. A number of the assessment components require that the course instructor and/or laboratory teaching assistant observe student groups as they conducted the actual experimentation. As the experimental work involves a good deal of coordination and teamwork, each group member is normally given the same score in these areas unless it is readily apparent that an individual student is contributing more or less than their fair share of the workload. Assessing experimental technique was used to help reinforce the notion that students are responsible for conducting a well-run experiment and that taking short cuts often lead to poor results and unacceptable, preventable errors. 
Table 1. Laboratory Exercise Assessment Scoring Rubric

( $\mathrm{G}=$ group assessment, all others based on individual performance)

\begin{tabular}{|l|l|l|l|l|l|}
\hline \multirow{2}{*}{ Element 1. Conduct Experiment } & \multicolumn{3}{|c|}{ Score } \\
\cline { 2 - 5 } & 1 & 2 & 3 & 4 & 5 \\
\hline configure system based on schematic diagram (G) & & & & & \\
\hline allow system to reach background steady state condition (G) & & & & & \\
\hline run experiment long enough to collect needed data (G) & & & & & \\
\hline document changes in system appearance during testing (G) & & & & & \\
\hline carefully gather data for flow rate determination (G) & & & & \\
\hline $\begin{array}{l}\text { Scoring: } 1 \text { = needs significant help/reminders } \\
3=\text { needs some help or occasional reminder } \\
5=\text { completes work independently }\end{array}$ & & & \\
\hline
\end{tabular}

\begin{tabular}{|l|l|l|l|l|c|}
\hline \multirow{2}{*}{ Element 2. Analyze and Interpret Data } & \multicolumn{3}{|c|}{ Score } \\
\cline { 2 - 5 } & 1 & 2 & 3 & 4 & 5 \\
\hline select suitable strategy for comparing results with theory & & & & & \\
\hline provide a clear, easily understandable comparison & & & & & \\
\hline identify differences between experimental results and theory & & & & & \\
\hline identify and quantify potential sources of error & & & & & \\
\hline discuss reasonableness of differences & & & & \\
\hline explain how differences could have been minimized & & & & & \\
\hline $\begin{array}{l}\text { Scoring: } 1=\text { little understanding of concept and/or little detail provided } \\
3=\text { some understanding of concept and/or little detail provided } \\
5\end{array}$ full understanding of concept and sufficient detail provided & & \\
\hline
\end{tabular}

\begin{tabular}{|l|l|l|l|l|l|}
\hline \multirow{2}{*}{ Element 3. Use Modern Engineering Tools } & \multicolumn{3}{|c|}{ Score } \\
\cline { 2 - 5 } & 1 & 2 & 3 & 4 & 5 \\
\hline properly configure the data acquisition system (G) & & & & & \\
\hline collect and save data in an appropriate file format (G) & & & & & \\
\hline set up a well-documented spreadsheet program & & & & & \\
\hline use proper spreadsheet functions to reduce data & & & & \\
\hline provide correct final values for needed/requested parameters & & & & & \\
\hline $\begin{array}{l}\text { Scoring: } 1=\text { improper/inappropriate/incorrect use of resource (major errors in use) } \\
3=\text { mostly proper/appropriate/correct use of resource (minor errors in use) }\end{array}$ \\
\begin{tabular}{l}
$5=$ completely proper/appropriate/correct use of resource (no errors in use) \\
\hline
\end{tabular}
\end{tabular}


Results gathered so far indicate that the sensor-based dye measurement technology and the computer-based data acquisition system (or the initially employed multimeter-based data acquisition system) have significantly improved student understanding of concepts related to ideal (and non-ideal) mixing. Related assessment results collected both before and after the dye tracer experiment was introduced are detailed in Table 2. Based on student feedback, much of this increased understanding is related to their ability to visually observe changes in the dye tracer concentration in the test system as the experiment proceeds. Students also positively responded to the simplicity and flexibility of the data acquisition system in allowing them to collect data over extended period of time and easily import this information into a spreadsheet program for subsequent analysis. A noticeable improvement in student work occurred between the first and second iterations of the experiment. This improvement was attributed, in large part, to inclusion of the assessment rubric as part of the lab handout. After inclusion of the rubric in the lab handout, there was a significant increase in the number of questions asked by students during the pre-lab discussion period. Written laboratory reports submitted for the experiment also demonstrably improved in quality after students had access to the rubric. Many students, however, have continued to struggle with the error analysis component of the experiment as they tend to focus more on generalities rather than specific and potentially quantifiable sources of error. Since differences between theoretical and actual experimental results have typically been less than 2.5 percent, error analysis has not appeared to be a major area of concern for students.

Table 2. Pre- and Post-Experiment Implementation Assessment Data

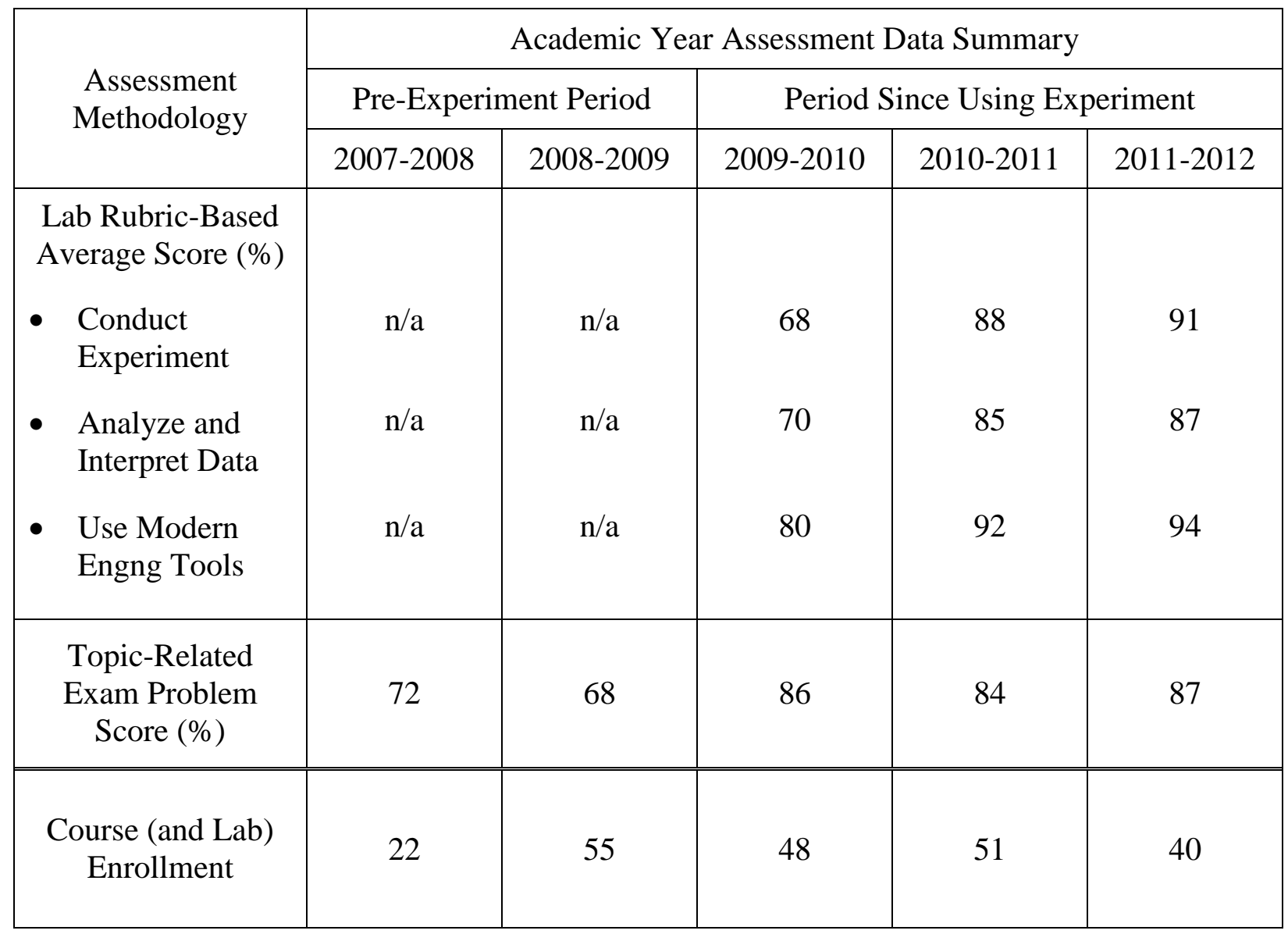


In addition to direct assessment of student laboratory work, scores on a related exam problem also have been used to assess the impact of the experimental work on student learning. As indicated in Table 2, scoring on the topic-related midterm or final exam problem has increased approximately 20 percent in the three years since the experiment was first implemented. It should be noted here that the dye tracer experiment replaced an experiment that made use of colorimetric-based analytical techniques. Since another course experiment still involves using colorimetric-based analytical techniques, there was little or no loss in conceptual content associated with the addition of the new experiment.

\section{Conclusions}

The use of a sensor-based system for dye tracer concentration tracking, together with a companion data acquisition system, has been successfully used to enhance student understanding of reactor mixing properties. The rubric developed for assessing student work, particularly when given to students ahead of the experimental work, has had a significant positive effect on the quality of student work and the level/depth of student understanding.

Applications for the use of the sensor and data acquisition system continue to be explored for other reactor systems. A better in-line dye injection system is being investigated to allow testing of a long, coiled section of tubing to be used as a plug flow-type reactor. Improvement of the inline dye injection system would also allow the use of a syringe pump to be used to provide a step increase in influent dye concentration and provide an alternative strategy for assessing reactor system mixing characteristics. The use of more complex test reactor systems would also allow the potential for the application of more complex mathematical modeling techniques to possibly include addressing student learning outcomes related to the application of mathematics, science and engineering principles (ABET Student Outcome 3a). In this and similar ways, the flexibility of the dye-based sensor and companion data acquisition system can be applied to a applied to a variety of engineering disciplines in laboratory exercises that can be associated with course work ranging from introductory-level courses to those at the graduate level.

\section{Bibliographic Information}

1. Viessman, W., Hammer, M.J., Perez, E.M., and Chadik, P.A., Water Supply and Pollution Control, $8^{\text {th }}$ edition, Prentice Hall, 2008.

2. Levenspiel, O., Chemical Reaction Engineering, $2^{\text {nd }}$ edition, John Wiley \& Sons, 1972. 